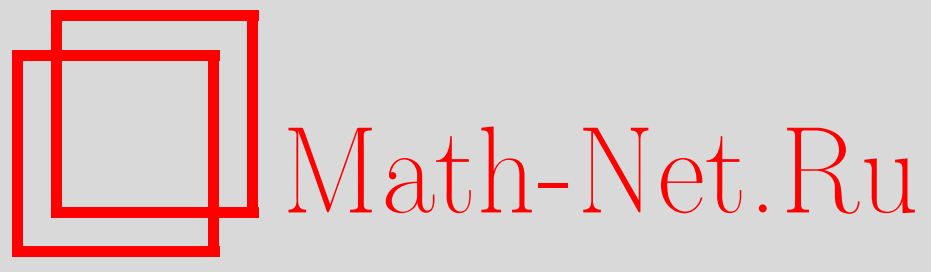

Е. М. Маслов, Л. А. Калякин, А. Г. Шагалов, Резонансный захват фазы бризера внешним возмущением, $T M \Phi$, 2007, том 152, номер 2, 356-367

DOI: https://doi.org/10.4213/tmf6092

Использование Общероссийского математического портала Math-Net.Ru подразумевает, что вы прочитали и согласны с пользовательским соглашением http://www . mathnet.ru/rus/agreement

Параметры загрузки:

IP: 3.89 .197 .203

26 апреля 2023 г., 02:33:04

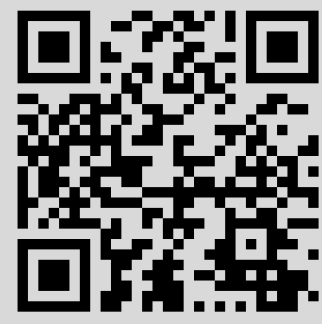




\title{
РЕЗОНАНСНЫЙ ЗАХВАТ ФАЗЫ БРИЗЕРА ВНЕШНИМ ВОЗМУЩЕНИЕМ
}

\begin{abstract}
Исследуется эффект резонансного захвата фазы в задаче о воздействии на бризер уравнения синус-Гордон малой осциллирующей внешней силы с медленно изменяющейся частотой. Получены уравнения, определяющие эволюцию во времени параметров возмущенного бризера. Описана регулярная асимптотическая процедура усреднения таких уравнений. Показано, что уже в главном порядке усредненные уравнения хорошо описывают явление резонансного захвата фазы, при котором происходит сильная раскачка колебаний бризера. Найдены необходимые и достаточные условия захвата, связывающие скорость изменения частоты возмущения и его амплитуду с начальными данными бризера.
\end{abstract}

Ключевые слова: уравнение синус-Гордон, бризер, захват фазы, теория возмущений, асимптотика, усреднение.

\section{1. ВВЕДЕНИЕ}

Большинство нелинейных волновых уравнений обладают широким набором точных периодических во времени решений, описывающих как локализованные возбуждения, так и распространяющиеся волны конечной амплитуды в различных физических системах. Существенным для практики является возможность селективного воздействия на те или иные периодические решения с помощью малых возмущений, включенных в уравнение, что позволяет контролируемым образом изменять амплитудно-фазовые характеристики таких решений. Один из перспективных методов в этом направлении основан на использовании явления резонансного захвата фазы (или, по другой терминологии, авторезонанса). Этот метод успешно применялся для генерации как простых кноидальных волн [1], так и многофазных решений [2] в периодической задаче для уравнения Кортевега-де Фриза, многофазных волн в нелинейном уравнении Шредингера [3], плазменных колебаний в уравнении

${ }^{*}$ Институт земного магнетизма, ионосферы и распространения радиоволн им. Н. В. Пушкова РАН, Московская обл., Троицк, Россия. E-mail: zheka@izmiran.ru

${ }^{\dagger}$ Институт математики Уфимского научного центра РАН, Уфа, Россия. E-mail: klenru@mail.ru

${ }^{\ddagger}$ Институт физики металлов УрО РАН, Екатеринбург, Россия. E-mail: shagalov@imp.uran.ru 
синус-Гордон (СГ) [4]. Идея метода восходит к работам Векслера и Мак-Миллана [5] по ускорению релятивистских частиц с использованием эффекта автофазировки. Адиабатическая теория захвата фазы для одномерного нелинейного осциллятора, возбуждаемого внешней силой с медленно изменяющейся частотой, была предложена Чириковым [6]. Современные исследования по асимптотической теории этого явления содержатся в работах [7].

Резонансный захват фазы в нелинейном осцилляторе возникает, если амплитуда возбуждения превышает некоторое критическое значение, зависящее от скорости изменения частоты. При этом частота колебаний осциллятора начинает автоматически подстраиваться к частоте накачки. Поскольку в нелинейной системе частота колебаний зависит от амплитуды, то при вариациях частоты внешней накачки будет изменяться и амплитуда захваченных колебаний, т.е. будет происходить эффективный обмен энергией между системой и накачкой. В частности, для нелинейного маятника медленное уменьшение частоты может привести к раскачке колебаний до амплитуд порядка единицы даже при очень малой амплитуде возбуждающей силы. Вместе с тем такой процесс может быть использован и для управления уже существующими колебаниями большой амплитуды, поскольку захват фазы внешним воздействием обеспечивает полный контроль как над амплитудой, так и над фазой колебаний. В качестве параметра управления в данном случае выступает частота внешней накачки.

В настоящей работе мы исследуем захват фазы бризера конечной амплитуды в уравнении СГ с пространственно-однородной квазипериодической малой накачкой:

$$
u_{t t}-u_{x x}+\sin u=\varepsilon \cos \psi(t), \quad 0<\varepsilon \ll 1 .
$$

Предполагается, что частота накачки $\Omega=\dot{\psi}$ медленно изменяется со временем: $\dot{\Omega}=O(\alpha),|\alpha| \ll 1$. В отсутствие возмущения (при $\varepsilon=0$ ) уравнение (1) допускает локализованное решение в виде бризера

$$
u=\phi_{\mathrm{b}}(x, t)=-4 \operatorname{arctg}\left(\frac{\varkappa_{0} \cos \left(\omega_{0} t+\theta_{0}\right)}{\omega_{0} \operatorname{ch}\left[\varkappa_{0}\left(x-x_{0}\right)\right]}\right), \quad \omega_{0}^{2}=1-\varkappa_{0}^{2},
$$

которое характеризуется двумя константами - $\varkappa_{0}$ и $\theta_{0}$. Координата центра бризера $x_{0}$ не играет роли в нашей задаче ввиду инвариантности уравнения (1) относительно сдвигов по $x$. Включение возмущения приводит к возбуждению в системе малых пространственно-однородных фоновых колебаний, которые взаимодействуют с бризером. Это взаимодействие приводит к тому, что в некоторой области значений параметров возмущения $\varepsilon$ и $\alpha$ и при некоторых условиях для начальных данных $\varkappa_{0}, \theta_{0}$ частота колебаний бризера начинает следовать за изменяющейся частотой накачки $\Omega$. При этом энергия бризера растет или убывает в зависимости от знака $\dot{\Omega}$. В настоящей работе мы находим эти значения и эти условия, а также получаем систему уравнений, описывающую динамику захваченных колебаний вплоть до больших времен (порядка $\varepsilon^{-1}$ ). Для этого мы используем теорию возмущений, основанную на методе обратной задачи [8]-[10] (раздел 2), обобщенный метод усреднения Крылова-Боголюбова-Митропольского [11] (раздел 3), а также приближение нелинейного маятника [6] (раздел 4). 


\section{2. АДИАБАТИЧЕСКОЕ ПРИБЛИЖЕНИЕ}

Как отмечалось выше, под действием внешнего возмущения в системе возникают однородные фоновые колебания, $\chi(t) \sim \varepsilon$. Ввиду их малости можно считать, что они описываются линейным уравнением

$$
\ddot{\chi}+\chi=\varepsilon \cos \psi(t) .
$$

Поэтому, полагая в (1)

$$
u(x, t)=\phi(x, t)+\chi(t),
$$

где $\phi(x, t) \rightarrow 0,|x| \rightarrow \infty$, приходим к возмущенному уравнению СГ вида [9]

$$
\phi_{t t}-\phi_{x x}+\sin \phi=\chi(t)(1-\cos \phi),
$$

где мы пренебрегли членами порядка $\varepsilon^{2}$. Это уравнение и описывает динамику локализованного бризера, находящегося под действием однородных фоновых колебаний. В дальнейшем мы будем считать, что в системе отсутствуют собственные линейные колебания, а частота внешнего возбуждения $\Omega$ не слишком близка к единице. Тогда для простоты в правой части уравнения (5) можно положить

$$
\chi(t)=\frac{\varepsilon \cos \psi}{1-\Omega^{2}} .
$$

Нетрудно проверить, что это выражение удовлетворяет уравнению (3) с ошибкой порядка $O(\alpha \varepsilon)$.

Применим теперь к уравнению (5) теорию возмущений, основанную на методе обратной задачи. При этом мы будем следовать работе [10], где были получены уравнения, определяющие эволюцию параметров бризера под действием произвольного возмущения. Согласно этому подходу решение, описывающее возмущенный бризер, представляется в виде

$$
\phi(x, t)=\phi_{\mathrm{b}}(x, t)+O(\varepsilon),
$$

где

$$
\phi_{\mathrm{b}}(x, t)=-4 \operatorname{arctg}\left(\frac{\varkappa(t) \cos \theta(t)}{\omega(t) \operatorname{ch}\left[\varkappa(t)\left(x-x_{0}\right)\right]}\right), \quad \omega^{2}=1-\varkappa^{2} .
$$

Здесь $\theta$ - фаза бризера, а $\omega$ и $\varkappa^{-1}$ - его характерные частота и размер $(0<\omega<1$, $0<\varkappa<1$ ). Параметризация

$$
\omega=\cos \gamma, \quad \varkappa=\sin \gamma, \quad 0<\gamma<\frac{\pi}{2},
$$

определяет характерную амплитуду бризера $\gamma=\operatorname{arctg}(\varkappa / \omega)$. Уравнения для этих параметров находятся в любом порядке по $\varepsilon$ последовательными приближениями из уравнений для данных рассеяния, отвечающих дискретному спектру в ассоциированной задаче рассеяния. Поправочные члены $O(\varepsilon)$ в формуле (7) связаны с непрерывной частью спектра. Они описывают искажение формы бризера на масштабе $\varkappa^{-1}$ и излучение, которое начинает распространяться от бризера после включения возмущения. В настоящей работе мы рассматриваем только первый член 
в уравнении (7). Такое приближение обычно называется адиабатическим. Ниже мы покажем, что этого приближения вполне достаточно для описания захвата фазы с высокой степенью точности.

Рассматривая правую часть уравнения (5) как возмущение и вычислив соответствующие интегралы (см. работу [10]), приходим в первом порядке по $\varepsilon$ к следующей системе уравнений адиабатического приближения:

$$
\begin{aligned}
& \dot{\gamma}=\varepsilon \frac{F(\gamma, \theta)}{1-\Omega^{2}} \cos \psi, \\
& \dot{\theta}=\omega(\gamma)+\varepsilon \frac{G(\gamma, \theta)}{1-\Omega^{2}} \cos \psi,
\end{aligned}
$$

где

$$
\begin{aligned}
& F(\gamma, \theta)= \frac{\pi}{4} \varkappa^{2} \sin \theta \cos ^{2} \theta \frac{4 \omega^{2}+\varkappa^{2} \cos ^{2} \theta}{\left(1-\varkappa^{2} \sin ^{2} \theta\right)^{5 / 2}}, \\
& G(\gamma, \theta)=\frac{\pi \omega}{4 \varkappa^{2}}\left\{\frac{\omega^{2} \varkappa \cos \theta}{\left(1-\varkappa^{2} \sin ^{2} \theta\right)^{3 / 2}} \times\right. \\
&\left.\times\left[1+\frac{\varkappa^{2} \cos ^{2} \theta\left(4 \omega^{2}+\varkappa^{2} \cos ^{2} \theta\right)}{\omega^{4}\left(1-\varkappa^{2} \sin ^{2} \theta\right)}\right]-\operatorname{arcsh}\left(\frac{\varkappa}{\omega} \cos \theta\right)\right\},
\end{aligned}
$$

а $\omega$ и $\varkappa$ определяются амплитудой $\gamma$ согласно (9). Эти уравнения содержат две быстрые переменные - фазу бризера $\theta$ и заданную фазу внешнего возмущения $\psi$ - и две медленные переменные - амплитуду бризера $\gamma$ и заданную частоту возмущения $\Omega=\dot{\psi}$. Функции $F(\gamma, \theta)$ и $G(\gamma, \theta)$ периодичны по $\theta$ с периодом $2 \pi$. При этом $F(\gamma, \theta)$ ограничена, а $G(\gamma, \theta)$ имеет особенности при $\gamma=0, \pi / 2$. Мы будем предполагать, что амплитуда $\gamma$ не слишком близка к этим значениям, так что $G(\gamma, \theta)$ также считается ограниченной. Отметим, что уравнения (10)-(13) с точностью до обозначений совпадают с уравнениями, полученными в работе [9].

Захват фазы означает по определению, что разность $\delta=\theta-\psi$ есть медленно изменяющаяся ограниченная функция времени. В этом случае динамическая система

$$
\begin{aligned}
& \dot{\gamma}=\varepsilon \frac{F(\gamma, \psi+\delta)}{1-\Omega^{2}} \cos \psi, \\
& \dot{\delta}=\Delta \omega(\gamma, \Omega)+\varepsilon \frac{G(\gamma, \psi+\delta)}{1-\Omega^{2}} \cos \psi, \\
& \dot{\psi}=\Omega,
\end{aligned}
$$

где

$$
\Delta \omega=\omega(\gamma)-\Omega, \quad \dot{\Omega}=O(\alpha),
$$

будет содержать только одну быструю переменную $\psi$. Такие решения могут существовать в системе (14)-(16) лишь при некоторых начальных условиях и при определенных ограничениях на $\alpha$ и $\varepsilon$. Это видно уже из того, что в режиме захвата расстройка $\Delta \omega$ должна быть мала, и эта малость должна сохраняться на всем временно́м интервале изменения $\Omega$, т.е. вплоть до $t \sim|\alpha|^{-1}$. Отсюда следует, во-первых, 
что $\omega(\gamma(0)) \approx \Omega(0)$ и, во-вторых, что скорость изменения $\Omega$ должна быть порядка средней (по фазе $\psi$ ) скорости изменения $\gamma$, т.е. $\alpha=O(\varepsilon)$. Поэтому мы положим

$$
\alpha=\beta \varepsilon, \quad|\beta|=O(1)
$$

где $\beta$ - некоторая фиксированная константа. Таким образом, малость $\Delta \omega$ зависит теперь только от малости $\varepsilon$ и от малости значения $\Delta \omega$ при $t=0$. Поскольку последняя величина никак не связана с $\varepsilon$, мы будем считать $\Delta \omega$ вторым независимым малым параметром, как это делал Гарифуллин [12] в задаче о нелинейном осцилляторе.

\section{3. УСРЕДНЕННЫЕ УРАВНЕНИЯ}

Учитывая все вышеизложенное, перейдем теперь к усредненному описанию процесса захвата фазы. При этом мы будем использовать обобщенный метод Крылова-Боголюбова-Митропольского (см., например, монографию [11]). В соответствии с этим подходом мы рассмотрим почти тождественное преобразование переменных $\gamma, \delta$ в новые "усредненные" переменные $\bar{\gamma}, \bar{\delta}$, записав его в форме асимптотического разложения

$$
\left(\begin{array}{l}
\gamma \\
\delta
\end{array}\right)=\left(\begin{array}{l}
\bar{\gamma} \\
\bar{\delta}
\end{array}\right)+\sum_{m=1}^{M-1} \sum_{n=0}^{N-1} \varepsilon^{m}(\overline{\Delta \omega})^{n}\left(\begin{array}{l}
u_{m n}(\bar{\gamma}, \bar{\delta}, \Omega, \psi) \\
v_{m n}(\bar{\gamma}, \bar{\delta}, \Omega, \psi)
\end{array}\right)+O\left(\varepsilon^{M}, \varepsilon(\overline{\Delta \omega})^{N}\right)
$$

Здесь в качестве второго независимого малого параметра мы использовали вместо $\Delta \omega$ величину $\overline{\Delta \omega}=\Delta \omega+O(\varepsilon)$, определяемую как

$$
\overline{\Delta \omega}=\omega(\bar{\gamma})-\Omega
$$

Потребуем теперь, чтобы скорость изменения медленных переменных $\bar{\gamma}$ и $\bar{\delta}$ не зависела от быстрой переменной $\psi$, т.е. положим

$$
\frac{d}{d t}\left(\begin{array}{c}
\bar{\gamma} \\
\bar{\delta}
\end{array}\right)=\left(\frac{0}{\Delta \omega}\right)+\sum_{m=1}^{M-1} \sum_{n=0}^{N-1} \varepsilon^{m}(\overline{\Delta \omega})^{n}\left(\begin{array}{c}
A_{m n}(\bar{\gamma}, \bar{\delta}, \Omega) \\
B_{m n}(\bar{\gamma}, \bar{\delta}, \Omega)
\end{array}\right)+O\left(\varepsilon^{M}, \varepsilon(\overline{\Delta \omega})^{N}\right)
$$

Таким образом, процедура усреднения системы (14)-(16) сводится к построению асимптотических разложений $(19),(21)$, т.е. к нахождению величин $u_{m n}, v_{m n}, A_{m n}$ и $B_{m n}$. Заметим, что в этих разложениях отсутствуют члены с $m=0$, поскольку при $\varepsilon=0$ система становится невозмущенной.

Подставим теперь (19), (21) в уравнения (14)-(16) и сравним коэффициенты при одинаковых $\varepsilon^{m}(\overline{\Delta \omega})^{n}$. При этом мы выберем для определенности линейный закон изменения $\Omega(t)$, положив $\dot{\Omega}=\alpha$, учтем выражения $(18),(20)$ и используем разложение

$$
\frac{d \overline{\Delta \omega}}{d t}=\varepsilon\left(A_{10} \omega^{\prime}(\bar{\gamma})-\beta\right)+\varepsilon \overline{\Delta \omega} A_{11} \omega^{\prime}(\bar{\gamma})+\varepsilon^{2} A_{20} \omega^{\prime}(\bar{\gamma})+\cdots
$$

В результате получается система рекуррентных дифференциальных уравнений первого порядка (по быстрой переменной $\psi$ ) для функций $u_{m n}$ и $v_{m n}$. Выражения 
для $A_{m n}$ и $B_{m n}$ находятся из условий ограниченности $u_{m n}$ и $v_{m n}$ по $\psi$. При этом решения $u_{m n}$ и $v_{m n}$ будут $2 \pi$-периодическими функциями быстрой переменной $\psi$, определенными с точностью до произвольных функций медленных переменных $\bar{\gamma}$, $\bar{\delta}, \Omega$. Выбор этих функций не влияет на точность и диктуется только соображениями удобства (см. монографию [11]). Мы выберем их таким образом, чтобы средние решений $u_{m n}$ и $v_{m n}$ по $\psi$ обращались в нуль. В этом случае величины $\bar{\gamma}$ и $\bar{\delta}$ приобретают естественный смысл средних значений от $\gamma$ и $\delta$. Кроме того, при таком выборе значительно упрощаются выражения для $A_{m n}$ и $B_{m n}$. Так, например, $A_{10}$ и $B_{10}$ находятся просто с помощью вычисления средних по $\psi$ в правых частях уравнений $(14),(15)$, а $A_{11}$ и $B_{11}$ обращаются в нуль. Таким образом, задача нахождения решений системы уравнений $(10),(11)$ в режиме резонансного захвата фазы сводится к интегрированию системы (21), которую обычно называют усредненной.

Описанная процедура позволяет получить усредненную систему с любой конечной степенью точности по двум малым параметрам - $\varepsilon$ и $\overline{\Delta \omega}$. Для систем с быстро вращающейся фазой, содержащих только один малый параметр, было показано [13], что предельная ошибка, остающаяся при использовании подобных процедур усреднения, экспоненциально мала по обратному малому параметру. В связи с этим необходимо отметить, что в контексте нашей исходной задачи учет в усредненных уравнениях членов с порядками малости выше $O(\varepsilon)$ является превышением точности, поскольку в решении (7) мы ограничиваемся анализом только первого адиабатического члена, параметры которого удовлетворяют уравнениям (10), (11), где отброшены члены порядка $\varepsilon^{2}$. K тому же и уравнения (5), (6) сами являются приближенными с той же степенью точности. По этим же причинам мы не анализируем здесь поправки $u_{m n}$ и $v_{m n}$; нам достаточно быть уверенными, что они существуют и являются ограниченными функциями времени. Такой подход, называемый обычно адиабатическим, является типичным в задачах подобного рода (см., например, работу [10]).

Принимая все это во внимание, мы сохраним в усредненных уравнениях (21) только главные члены, определяемые коэффициентами $A_{10}$ и $B_{10}$. Вычисляя их с использованием конкретных выражений (12), (13), получаем усредненную систему первого порядка по $\varepsilon$ и $\overline{\Delta \omega}$ в виде

$$
\begin{aligned}
& \frac{d \bar{\gamma}}{d t}=\varepsilon \frac{g(\bar{\gamma})}{1-\Omega^{2}(t)} \sin \bar{\delta} \\
& \frac{d \bar{\delta}}{d t}=\overline{\Delta \omega}+\varepsilon \frac{g^{\prime}(\bar{\gamma})}{1-\Omega^{2}(t)} \cos \bar{\delta}
\end{aligned}
$$

Здесь

$$
g(\bar{\gamma})=\frac{1}{2}[K(\bar{\varkappa})-E(\bar{\varkappa})] \geqslant 0, \quad g^{\prime}(\bar{\gamma})=\frac{1}{2} E(\bar{\varkappa}) \operatorname{tg} \bar{\gamma} \geqslant 0
$$

$K(\bar{\varkappa})$ и $E(\bar{\varkappa})$ суть полные эллиптические интегралы первого и второго рода, соответственно, $\bar{\varkappa}=\sin \bar{\gamma}, \Omega(t)=\Omega_{0}+\alpha t$, а $\overline{\Delta \omega}$ определено выражением (20). Режиму захвата соответствуют решения с ограниченными функциями $\bar{\delta}(t)$. Отметим, что 

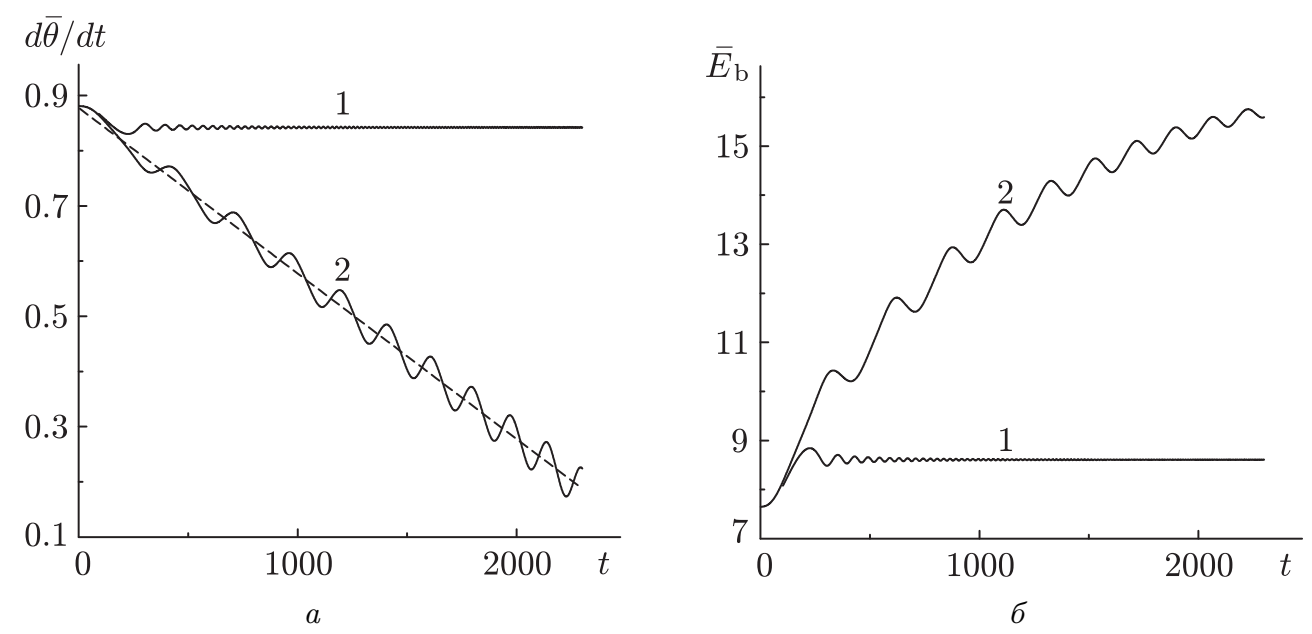

Рис. 1. Зависимость от времени средних частоты (а) и энергии (б) бризера. Кривые 1 - отсутствие захвата, $\varepsilon=0.0016$. Кривые 2 - режим захвата, $\varepsilon=$ 0.0019 . Пунктирная линия - частота $\Omega(t)=\Omega_{0}+\alpha t$, где $\Omega_{0}=\cos \bar{\gamma}(0) \simeq 0.8776$, $\alpha=-0.0003$.

полученная система имеет гамильтониан

$$
H(\bar{\gamma}, \bar{\delta} ; \Omega)=\bar{\gamma} \Omega-\sin \bar{\gamma}-\varepsilon \frac{g(\bar{\gamma})}{1-\Omega^{2}} \cos \bar{\delta}
$$

На рис. 1 приведены зависимости от времени средних частоты и энергии бризера, определенных соответственно как $d \bar{\theta} / d t=\Omega(t)+d \bar{\delta} / d t, \bar{E}_{\mathrm{b}}=16 \sin \bar{\gamma}$, найденные с помощью численного интегрирования системы $(23),(24)$ с начальными условиями $\bar{\gamma}(0)=0.5, \bar{\delta}(0)=0, \overline{\Delta \omega}(0)=0$. При каждом фиксированном $\alpha$ переход в режим захвата наблюдался при превышении $\varepsilon$ некоторого критического значения. В частности, при выбранных начальных условиях и $\alpha=-0.0003$ мы получили $\varepsilon_{\mathrm{cr}}=0.00178$.

Для проверки мы численно проинтегрировали исходное уравнение СГ (1) с начальными условиями $u(x, 0)=\phi_{\mathrm{b}}(x, 0)+\chi(0), u_{t}(x, 0)=0$. При этом для параметров бризера и возмущения брались те же начальные условия, что и для системы $(23),(24)$. Частота бризера определялась по моментам прохождения поля $u(x, t)$ через нуль, а энергия - путем интегрирования плотности энергии по ширине бризера.

Сравнение полученных результатов показало, что соответствующие кривые практически совпадают, так что на рис. 1 они были бы неразличимы. Отметим лишь, что истинное значение $\varepsilon_{\mathrm{cr}}$, следующее из решения уравнения (1), составило 0.00179 .

\section{4. ПРИБЛИЖЕНИЕ НЕЛИНЕЙНОГО МАЯТНИКА}

Уравнения (23), (24) описывают динамику средних значений $\gamma$ и $\delta$ на большом временно́м интервале $0 \leqslant t \lesssim \varepsilon^{-1}$. Однако, как видно из рис. 1 , сам захват происходит на начальных временах, почти сразу после включения возмущения. Этим обстоятельством, следуя работе [6], мы и воспользуемся при определении условий 
захвата. Для этого перейдем от системы (23), (24) к уравнению второго порядка для $\bar{\delta}$. Дифференцируя уравнение (24) и используя (23), находим

$$
\frac{d^{2} \bar{\delta}}{d t^{2}}=\varepsilon \frac{g(\bar{\gamma}) \omega^{\prime}(\bar{\gamma})-g^{\prime}(\bar{\gamma}) \overline{\Delta \omega}}{1-\Omega^{2}(t)} \sin \bar{\delta}-\varepsilon \beta+O\left(\varepsilon^{2}, \varepsilon(\overline{\Delta \omega})^{2}\right) .
$$

Здесь мы учли, что, поскольку $A_{11}=B_{11}=0$, то ошибка в правых частях уравнений $(23),(24)$ имеет порядок лишь $O\left(\varepsilon^{2}, \varepsilon(\overline{\Delta \omega})^{2}\right)$, и поэтому сохранили в уравнении $(27)$ член порядка $O(\varepsilon \overline{\Delta \omega})$, получающийся после дифференцирования второго члена в правой части (24).

Рассмотрим теперь начальный временно́й интервал $0 \leqslant t \ll \varepsilon^{-1}$, когда, собственно, и происходит захват фазы. В этом случае в правой части уравнения (27) можно пренебречь зависимостью от времени коэффициента при $\sin \bar{\delta}$, взяв его значение, например, при $t=0$. Таким образом, на рассматриваемом временно́м масштабе мы приходим к уравнению нелинейного маятника

$$
\frac{d^{2} \bar{\delta}}{d \tau^{2}}=\lambda \sin \bar{\delta}-\beta, \quad 0 \leqslant \tau \ll \varepsilon^{-1 / 2},
$$

где $\tau=\varepsilon^{1 / 2} t$ - медленное время, а константа $\lambda$ с необходимой степенью точности вычисляется по начальным значениям $\gamma=\bar{\gamma}+O(\varepsilon)$ и $\Omega$ :

$$
\lambda \simeq \frac{g(\gamma(0)) \omega^{\prime}(\gamma(0))-g^{\prime}(\gamma(0)) \Delta \omega(0)}{1-\Omega_{0}^{2}} .
$$

В частности, если начальная расстройка равна нулю или достаточно мала, $\Delta \omega(0) \lesssim$ $O(\varepsilon)$, то

$$
\lambda \simeq-\frac{g(\gamma(0))}{\sin \gamma(0)} .
$$

Заметим, что $\lambda$ всегда отрицательна.

Общие свойства всех возможных решений уравнения (28) можно получить, проанализировав различные фазовые траектории, определяемые первым интегралом (энергией)

$$
E\left(\bar{\delta}, \bar{\delta}_{\tau}\right)=\frac{1}{2} \bar{\delta}_{\tau}^{2}+V(\bar{\delta}), \quad V(\bar{\delta})=\lambda \cos \bar{\delta}+\beta \bar{\delta} .
$$

Ясно, что фазовый портрет существенно зависит от соотношения параметров $\beta$ и $\lambda$.

В случае, когда $|\beta / \lambda|>1$, неподвижных точек нет. Фазовые траектории представляют собой периодически деформированные параболы (рис. 2а). При любых начальных условиях все решения быстро растут со временем: $\bar{\delta}(\tau)=-\beta \tau^{2} / 2+O(\tau)$, $\tau \rightarrow \pm \infty$. Поэтому захват фазы невозможен, если частота накачки меняется слишком быстро по сравнению с величиной ее амплитуды. Если же частота накачки меняется достаточно медленно, так что

$$
\left|\frac{\beta}{\lambda}\right|<1
$$

то на фазовом портрете возникают две бесконечные последовательности неподвижных точек, устойчивых и неустойчивых (рис. 2б). Из каждой неустойчивой точки 

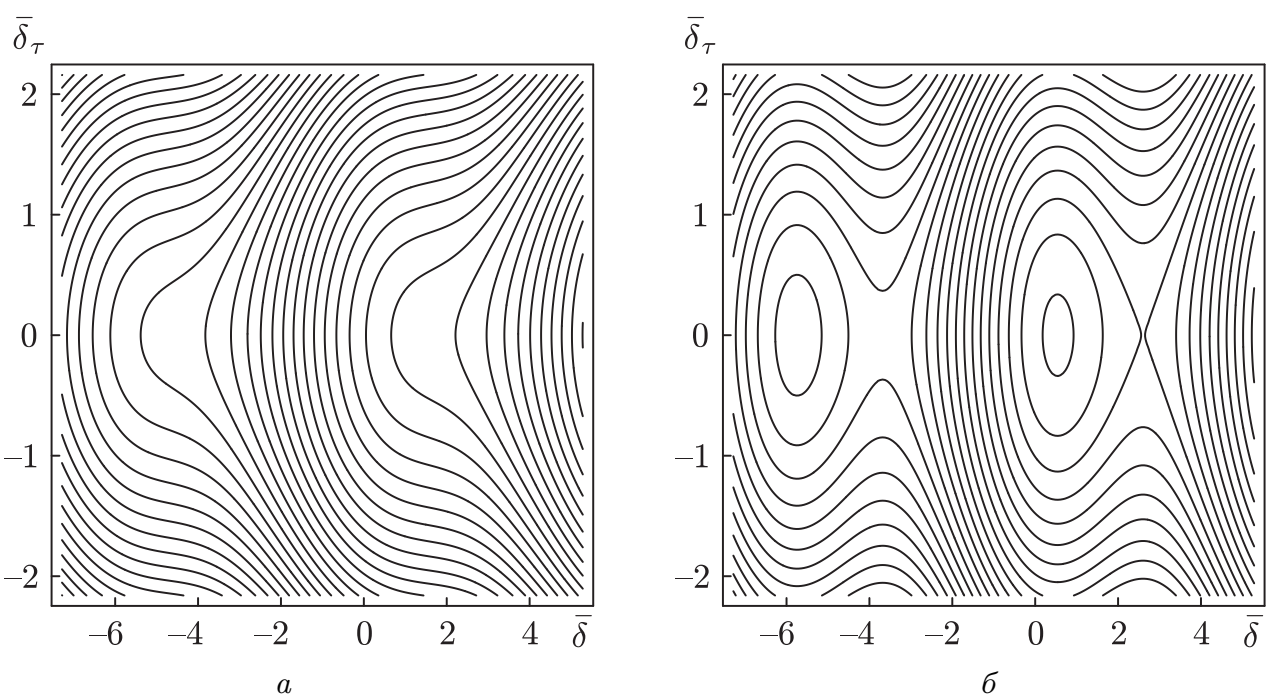

Рис. 2. Фазовый портрет уравнения (28): $|\beta / \lambda|>1$ (a), $|\beta / \lambda|<1$ (б).

выходит пара сепаратрис, которые уходят на бесконечность, а также сепаратрисная петля, охватывающая соседнюю устойчивую точку. Замкнутые траектории внутри петли соответствуют периодическим решениям, т.е. режиму захвата. Для таких решений амплитуда бризера будет в среднем расти, если $\beta<0$, или убывать, если $\beta>0$. В этом можно убедиться, подставив в уравнение $(23) \sin \bar{\delta}$ из уравнения (28) и усреднив $(23)$ по колебаниям $\bar{\delta}(t)$.

Очевидно, что неравенство (32) является необходимым, но не достаточным условием захвата. Необходимо также, чтобы начальная точка $\left(\bar{\delta}(0), \bar{\delta}_{\tau}(0)\right)$ находилась внутри одной из сепаратрисных петель. Пусть $\bar{\delta}_{n}^{(1)}$ и $\bar{\delta}_{n}^{(2)}$ суть минимальное и максимальное значения $\bar{\delta}$ на $n$-й сепаратрисной петле, а $\bar{\delta}_{n}^{(0)}-$ значение в устойчивой точке внутри этой петли: $\bar{\delta}_{n}^{(1)}<\bar{\delta}_{n}^{(0)}<\bar{\delta}_{n}^{(2)}$. Тогда для периодичности $\bar{\delta}(\tau)$ необходимо и достаточно, чтобы для некоторого $n$ выполнялись неравенства

$$
\begin{gathered}
\bar{\delta}_{n}^{(1)}<\bar{\delta}(0)<\bar{\delta}_{n}^{(2)}, \\
E_{n}^{(0)} \leqslant E\left(\bar{\delta}(0), \bar{\delta}_{\tau}(0)\right)<E_{n}^{(\mathrm{s})},
\end{gathered}
$$

где $E_{n}^{(\mathrm{s})}$ и $E_{n}^{(0)}$ - значения первого интеграла на $n$-й сепаратрисной петле и в соответствующей устойчивой точке: $E_{n}^{(\mathrm{s})}=V\left(\bar{\delta}_{n}^{(1)}\right)=V\left(\bar{\delta}_{n}^{(2)}\right), E_{n}^{(0)}=V\left(\bar{\delta}_{n}^{(0)}\right)$. С учетом того, что $\lambda<0$, величины $\bar{\delta}_{n}^{(i)}, i=0,1,2, n=0, \pm 1, \pm 2, \ldots$, определяются следующим образом:

1) при $\beta<0$

$$
\bar{\delta}_{n}^{(0)}=2 n \pi+\arcsin \frac{\beta}{\lambda}, \quad \bar{\delta}_{n}^{(2)}=(2 n+1) \pi-\arcsin \frac{\beta}{\lambda},
$$


$\bar{\delta}_{n}^{(1)}$ есть корень уравнения

$$
\cos \delta+\frac{\beta}{\lambda} \delta=-\sqrt{1-\left(\frac{\beta}{\lambda}\right)^{2}}+\frac{\beta}{\lambda}\left[(2 n+1) \pi-\arcsin \frac{\beta}{\lambda}\right],
$$

лежащий в интервале $\bar{\delta}_{n-1}^{(2)}<\bar{\delta}_{n}^{(1)}<\bar{\delta}_{n}^{(0)}$;

2) при $\beta>0$

$$
\bar{\delta}_{n}^{(0)}=(2 n+2) \pi+\arcsin \frac{\beta}{\lambda}, \quad \bar{\delta}_{n}^{(1)}=(2 n+1) \pi-\arcsin \frac{\beta}{\lambda},
$$

$\bar{\delta}_{n}^{(2)}$ есть корень уравнения (36), лежащий в интервале $\bar{\delta}_{n}^{(0)}<\bar{\delta}_{n}^{(2)}<\bar{\delta}_{n+1}^{(1)}$.

Итак, неравенства (32)-(34) являются необходимыми и достаточными условиями захвата фазы бризера внешним возмущением. Эти неравенства означают, что фазовый портрет уравнения (28) имеет сепаратрисные петли, и что начальная точка $\left(\bar{\delta}(0), \bar{\delta}_{\tau}(0)\right)$ лежит внутри одной из этих петель. При $|\beta / \lambda| \rightarrow 0$ сепаратрисные петли расширяются, их размер вдоль оси $\bar{\delta}$ стремится к $2 \pi$. При $|\beta / \lambda| \rightarrow 1$ петли стягиваются к точкам $\operatorname{sgn}(\beta / \lambda) \pi / 2+2 n \pi$.

Предположим, что начальная точка находится внутри $n$-й сепаратрисной петли. Будем теперь медленно увеличивать скорость изменения частоты накачки. Критическое значение $\beta$, при котором произойдет срыв захвата, получается, когда стягивающаяся петля пересечет начальную точку. Таким образом, $\beta_{\mathrm{cr}}$ находится как корень уравнения

$$
E\left(\bar{\delta}(0), \bar{\delta}_{\tau}(0) ; \beta, \lambda\right)=E_{n}^{(\mathrm{s})}(\beta, \lambda) .
$$

Если начальная расстройка достаточно мала, $\Delta \omega(0)=\overline{\Delta \omega}(0)+O(\varepsilon)=o\left(\varepsilon^{1 / 2}\right)$, то в силу (24) в левой части уравнения (38) можно пренебречь кинетическим членом. В этом случае (38) приобретает вид уравнения $(36)$, где $\delta=\delta(0)=\bar{\delta}(0)+O(\varepsilon)$. Это уравнение теперь определяет в неявной форме $\beta_{\mathrm{cr}} / \lambda$ как функцию от начальной разности фаз $\delta(0)$.

Рассмотрим для примера случай, когда частота накачки уменьшается, т.е. $\beta<0$, начальная расстройка $\Delta \omega(0)=0$, а начальная разность фаз бризера и накачки распределена в интервале от 0 до $2 \pi$. При этом для $0 \leqslant \delta(0)<\pi$ в уравнении (36) следует брать $n=0$ (нулевая петля), а для $\pi<\delta(0) \leqslant 2 \pi-n=1$ (первая петля). При $\delta(0)=\pi$, очевидно, имеем $\beta_{\text {cr }} / \lambda=0$, т.е. в противофазе захвата не происходит никогда. При $\delta(0)=\pi / 2$ захват происходит при всех $\beta$ вплоть до предельно быстрого изменения частоты, когда $\beta=\lambda$. График зависимости $\beta_{\mathrm{cr}} / \lambda$ от $\delta(0)$ представлен на рис. 3. Режиму захвата отвечают значения $\beta / \lambda$ ниже сплошной кривой. На этом же рисунке изображены значения $\beta_{\mathrm{cr}} / \lambda$, найденные с помощью численного интегрирования уравнения СГ (1). Отметим, что в узкой области вблизи значения $\delta(0)=\pi$ мы наблюдали в численном эксперименте интенсивное излучение волн бризером и отсутствие захвата фазы даже при очень малых скоростях изменения частоты накачки. Ясно, что в этой области адиабатическое приближение становится неприменимым. Тем не менее, несмотря на это и на определенную грубость приближения нелинейного маятника, полученные результаты, как видно из рис. 3, 


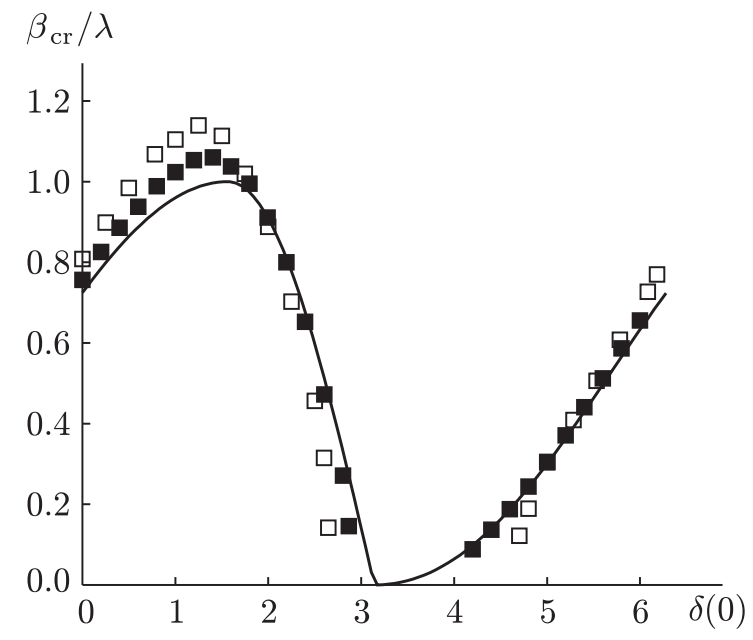

Рис. 3. Зависимость $\beta_{\mathrm{cr}} / \lambda$ от начальной разности фаз. Сплошная кривая получена при решении уравнения (36); $\square$ - значения, полученные путем численного интегрирования уравнения СГ при $\alpha=-0.0003 ; \mathbf{\square}$ - то же при $\alpha=-0.00003$.

достаточно хорошо согласуются с решением уравнения СГ путем численного интегрирования.

\section{5. ЗАКЛЮЧЕНИЕ}

В настоящей работе мы рассмотрели эффект резонансного захвата фазы в уравнении СГ на примере бризера, находящегося под действием малой осциллирующей внешней силы с медленно изменяющейся частотой. С помощью теории возмущений, основанной на методе обратной задачи, мы получили систему уравнений (14)-(16) для параметров бризера, описывающую эффект захвата в адиабатическом приближении. Особенность этой системы, относящейся к классу динамических систем с быстро вращающейся фазой, состоит в наличии двух независимых малых параметров - амплитуды внешней силы $\varepsilon$ и расстройки частоты $\Delta \omega$. Мы описали регулярную процедуру усреднения таких систем, основанную на асимптотических разложениях по обоим малым параметрам, и получили в явном виде усредненную систему первого приближения (23), (24), которая является гамильтоновой. Сравнение решений, полученных численным интегрированием этой системы, с соответствующими численными решениями исходного уравнения СГ показало совпадение результатов с высокой степенью точности. Для определения условий захвата и нахождения критических параметров возмущения мы использовали приближение нелинейного маятника (28). В итоге получены необходимые и достаточные условия захвата в виде неравенств (32)-(34), а также уравнение (38) для критических значений $\beta$, которое в случае малых начальных расстроек приобретает форму уравнения (36). Реше- 
ние этого уравнения хорошо согласуется с результатами, полученными с помощью численного интегрирования уравнения СГ.

Благодарности. Авторы признательны организаторам конференции "Нелинейная физика. Теория и эксперимент. IV" за возможность представить результаты этой работы в виде доклада. Авторы также благодарны участникам конференции, особенно Л. Фридланду и О. Киселеву, за полезные дискуссии. Работа выполнена при частичной поддержке INTAS (грант № 03-51-4286) и РФФИ (гранты №o 06-01-00124, 06-01-92052).

\section{Список литературы}

[1] I. Aranson, B. Meerson, T. Tatjima, Phys. Rev. A, 45 (1992), 7500.

[2] L. Friedland, A. G. Shagalov, Phys. Rev. Lett., 90 (2003), 074101.

[3] L. Friedland, A. G. Shagalov, Phys. Rev. E, 71 (2005), 036206.

[4] L. Friedland, Phys. Rev. E, 55 (1997), 1929.

[5] В. И. Векслер, ДАН СССР, 43 (1944), 346; E. M. MacMillan, Phys. Rev., 68 (1945), 143.

[6] Б. В. Чириков, ДАН СССР, 125 (1959), 1015.

[7] Л. А. Калякин, Докл. РАН, 378 (2001), 594; ТМФ, 133 (2002), 429.

[8] D. J. Kaup, A. C. Newell, Proc. Roy. Soc. London. Ser. A, 361 (1978), 413.

[9] M. J. Inoue, J. Phys. Soc. Japan, 47 (1979), 1723.

[10] В. И. Карпман, Е. М. Маслов, В. В. Соловьев, ЖЭТФ, 84 (1983), 289.

[11] Н. Н. Моисеев, Асимптотические методы нелинейной механики, Наука, М., 1981.

[12] Р. Н. Гарифуллин, ЖВМ и МФ, 46 (2006), 1605.

[13] А. И. Нейштадт, Прикл. матем. и мех., 48 (1984), 197. 